\title{
ASSEMBLY AND CHARACTERIZATION OF 8-ARM AND 12-ARM DNA BRANCHED JUNCTIONS
}

\author{
Xing Wang and Nadrian C. Seeman \\ Department of Chemistry, New York University, New York, NY 10003, USA \\ Xing Wang: xw246@nyu.edu; Nadrian C. Seeman: ned.seeman@nyu.edu
}

\begin{abstract}
Branched DNA molecules can be assembled into objects and networks directed by sticky-ended cohesion. The connectivity of these species is limited by the number of arms flanking the branch point. To date, the only branched junctions constructed contain six or fewer arms. We report the construction of DNA branched junctions that contain either 8 or 12 double helical arms surrounding a branch point. The design of the 8 -arm junction expoits the limits of a previous approach to thwart branch migration, but the design of the 12-arm junction uses a new to principle achieve this end. The 8 -arm junction is stable with 16 nucleotide pairs per arm, but the 12 -arm junction has been stabilized by 24 nucleotide pairs per arm. Ferguson analysis of these junctions in combination with three, four, five, and six-arm junctions indicates a linear increase in friction constant as the number of arms increases; the four-arm junction migrates anomalously at $4{ }^{\circ} \mathrm{C}$., suggesting stacking of its domains. All strands in both the 8-arm and 12-arm junctions show similar responses to hydroxyl radical autofootprinting analysis, indicating that they lack any dominant stacking structures. The stability of the 12-arm junction demonstrates that the number of arms in a junction is not limited to the case of having adjacent identical base pairs flanking the junction. The ability to construct eight-arm and twelve-arm junctions increases the number of objects, graphs and networks that can be built from branched DNA components. In principle, the stick structure corresponding to cubic close packing is now a possible target for assembly by DNA nanotechnology.
\end{abstract}

\section{Keywords}

Branched DNA Motifs; N-Connected DNA-Networks; N-Connected DNA Graphs; Hydroxyl Radical Autofootprinting; Gel Electrophoresis; Ferguson Plots

DNA branched junctions containing 3 or 4 arms around a single point are seen as ephemeral intermediates in the biological processes of replication ${ }^{1}$ and recombination. ${ }^{2}$ In addition, immobile versions of these junctions have served as the basis for virtually all of the work done in structural DNA nanotechnology. ${ }^{3}$ A variety of DNA structures and motifs have been created using these components, ${ }^{4}$ including stick figures whose edges are DNA double helices and whose vertices are the branch points of branched junctions; these include molecules with the connectivities of a cube ${ }^{5}$ and a truncated octahedron, ${ }^{6}$ as well as an irregular graph. ${ }^{7}$ The connectivity of DNA structures of this sort is limited by the number of double helical DNA arms that can flank a branch point.

Correspondence to: Nadrian C. Seeman, ned. seeman@nyu.edu.

Supporting Information Available. Sequences of junctions used for comparison with the reported junctions in Figure 3. 
Junctions containing dyad sequence symmetry are capable of branch migration, an isomerization that can relocate the branch point. ${ }^{8}$ To exclude dyad symmetry rigorously from a branched junction, one can only use once each of the four conventional base pairs to flank a junction. Thus, with four base pairs, one ought to be limited to 4 -arm junctions. However, if one places two identical base pairs adjacent to each other, it is not physically possible for the dinucleotide in the middle to pair with itself. This opens a larger range of junctions, of which the 5-arm and 6-arm junction have been constructed previously. ${ }^{9}$ This principle ought to be applicable to 8 -arm junctions, and we demonstrate here that it is.

However, some of the more interesting connected networks require 12-arm junctions for their assembly. Most prominent of these is the stick version of cubic close-packing, a facecentered cubic lattice, in which each unit is surrounded by 12 other units. Traditionally, these units are modeled in chemistry as balls, representing atoms. However, one can also make a stick version of this lattice, which is highly stable. This notion was elaborated by Buckminster Fuller, in his 'octahedral strut' structure, ${ }^{10}$ seen throughout the world in public buildings, such as the Javits Center in New York City and many airports, such as those in Lisbon, Portugal and in Chengdu, China. Thus, it is important to be able to construct 12connected networks, but there seems to be a conflict with the principles noted above.

Here, we demonstrate the validity of a new principle for designing junctions with more than eight branches; the basis of this approach is to distribute the four base pairs symmetrically about the center, thereby creating an entropic barrier to forming branch migratory structures. Thus, for a 12-arm branched junction, the sequence of base pairs flanking the junction could be G-C, T-A, C-G, A-T, G-C, T-A, C-G, A-T, G-C, T-A, C-G, A-T, for the junctionflanking base pair of arms 1 through 12 , respectively. The idea is that the loop entropy terms for a 12 component loop would be favored over an 8-component loop and a 4-component loop; the treatment of Cantor and Schimmel ${ }^{11}$ suggests a preference of about $1.6 \mathrm{Kcal} / \mathrm{mol}$ for the 12 component loop. We have used this method here, and we find that it is indeed possible to produce a 12-arm junction using this notion. We demonstrate formation of both the 8-arm and 12-arm junctions by gel electrophoresis, and we provide preliminary structural characterization by Ferguson plots and by hydroxyl radical autofootprinting. ${ }^{12} \mathrm{We}$ find that the arms are double helical, that the junctions display no stacking preferences, that there is no branch migration, and that the junctions behave as though their arms are roughly independent of each other. The analytical procedure is illustrated in Scheme 1.

\section{MATERIALS AND METHODS}

\section{Sequence Design}

All sequences have been assigned using the program SEQUIN. ${ }^{15}$ To design the 8 -arm junction, we have used the previously constructed 6-arm junction, JYL6G as a starting point. ${ }^{9}$ We have interrupted strands five and six of the junction to insert two more arms; thus, arms I, II, III, IV, V and VII are the same as in JYL6G, but arms VI and VIII have been designed for this work.

The 12-arm junction was designed by repeating the sequence G-C, T-A, C-G, and A-T three times counterclockwise to assign the junction-flanking base pairs. Instability indicated by non-denaturing gel electrophoresis led us to add eight nucleotide pairs to each arm of the 12arm junction, so as to stabilize it. The sequences of the 8 -arm and the 12-arm junctions are shown in Figure 1.

Nine other junctions are used as electrophoretic standards in this study, two three-arm junctions, JYG \& JYO, two four-arm junctions, JXG \& JXO, two five-arm junctions JYL5G \& JXW5O, and two six-arm junctions JYL6G \& JXW6O, and one more eight-arm junction 
JXW8O. JXG and JXO are two extensions of the well-characterized junction $\mathrm{J} 1 .{ }^{16}$ The last character of the name of each of these junctions indicates the number of nucleotide pairs per arm; we is am increasing alphabetic code related to, but longer than the familiar hexadecimal, where A would correspond to 11 , and $\mathrm{F}$ to 15 . The range is convenient for extending through the rest of the alphabet, $\mathrm{G}$ corresponds to 16 and $\mathrm{O}$ to 24 . The sequences are listed in the supplementary material.

\section{Synthesis and Purification of DNA}

All DNA molecules used in this study have been synthesized on an Applied Biosystems 394 automatic DNA synthesizer, removed from the support, and deprotected using routine phosphoramidite procedures. ${ }^{17}$ DNA strands have been purified by denaturing gel electrophoresis; bands were cut out of 10 to $20 \%$ denaturing gels and eluted in a solution (termed Elution Buffer) containing $500 \mathrm{mM}$ ammonium acetate, $10 \mathrm{mM}$ magnesium acetate, and $1 \mathrm{mM}$ EDTA. The eluates were subjected to extraction with $n$-butanol to remove the ethidium, followed by ethanol precipitation.

\section{Formation of Junction Molecules}

Junctions are formed by mixing a stoichiometric quantity of each strand, as estimated by $\mathrm{OD}_{260}$. The strands were dissolved to a concentration of $2 \mu \mathrm{M}$ in $20 \mu \mathrm{L}$ of a solution containing $40 \mathrm{mM}$ Tris (pH 8.0), $20 \mathrm{mM}$ acetic acid, $2 \mathrm{mM}$ EDTA, and $12.5 \mathrm{mM}$ magnesium acetate (TAEMg), heated to $90^{\circ} \mathrm{C}$ for $5 \mathrm{~min}$, and then cooled to $65^{\circ} \mathrm{C}$ for $15 \mathrm{~min}, 45^{\circ} \mathrm{C}$ for $15 \mathrm{~min}, 37^{\circ} \mathrm{C}$ for $20 \mathrm{~min}$, room temperature for $20 \mathrm{~min}$, and finally $4^{\circ} \mathrm{C}$ for $20 \mathrm{~min}$. Errors in stoichiometry are estimated by native gel electrophoresis of adjacent dimers. A single band on a native gel is taken to indicate a homogeneous stoichiometric complex.

\section{Hydroxyl Radical Analysis}

Individual strands of the junctions are radioactively labeled, and are additionally gel purified from a $10 \%$ denaturing polyacrylamide gel. Each of the labeled strands [approximately 10 pmol in $50 \mathrm{mM}$ Tris. $\mathrm{HCl}$ ( $\mathrm{pH} 7.5$ ) containing $10 \mathrm{mM} \mathrm{MgCl}_{2}$ ] is annealed to an excess of the unlabeled complementary strand or it is annealed to an excess of a mixture of the other strands in the motif, or it is left untreated as a control, or it is treated with sequencing reagents ${ }^{18}$ for a sizing ladder. The samples are annealed by heating to $90 \mathrm{C}$ for $3 \mathrm{~min}$ and then slowly cooled to $4{ }^{\circ} \mathrm{C}$. Hydroxyl radical cleavage of the double-strand and junction samples for all strands takes place at $4{ }^{\circ} \mathrm{C}$ for $2 \mathrm{~min},{ }^{19}$ with modifications noted by Churchill, et al. ${ }^{20}$ The reaction is stopped by addition of thiourea. The sample is dried, dissolved in a formamide/dye mixture, and loaded directly onto a $14 \%$ polyacrylamide/ $8.3 \mathrm{M}$ urea sequencing gel. Autoradiograms were quantitated using a Bio-Rad GS-250 Molecular Imager.

\section{Radioactive Phosphorylation}

$1 \mu \mathrm{g}$ of an individual strand of DNA is dissolved in $10 \mu \mathrm{L}$ of a solution containing $66 \mathrm{mM}$ Tris $\bullet \mathrm{HCl}$, pH 7.6, $1 \mathrm{mM}$ spermidine, $10.0 \mathrm{mM} \mathrm{MgCl} 2,15 \mathrm{mM}$ dithiothreitol (DTT), $0.2 \mathrm{mg} /$ $\mathrm{ml}$ nuclease free bovine serum albumin (BSA) (BRL), and mixed with $1-2 \mu \mathrm{L}$ of $1.25 \mathrm{mM}$

$\gamma^{-32} \mathrm{P}-\mathrm{ATP}(10 \mathrm{mCi} / \mathrm{ml})$ and $1-2$ units of polynucleotide kinase (USB) for $45 \mathrm{~min}-2 \mathrm{hrs}$ at 37

${ }^{\circ} \mathrm{C}$. The reaction is stopped by dessication or ethanol precipitation of DNA.

\section{Polyacrylamide Gel Electrophoresis}

Denaturing Gels-These gels contain $8.3 \mathrm{M}$ urea and are run at $55^{\circ} \mathrm{C}$. Gels contain 10 $20 \%$ acrylamide (19:1, acrylamide:bisacrylamide). The running buffer consists of $100 \mathrm{mM}$ Tris.HCl, pH 8.3, 89 mM Boric acid, 2 mM EDTA (TBE). The sample buffer consists of 10 $\mathrm{mM} \mathrm{NaOH}, 1$ mM EDTA, containing 0.1\% Xylene Cyanol FF tracking dye. Gels are run on 
an IBI Model STS 45 electrophoresis unit at 70 watts $(50 \mathrm{v} / \mathrm{cm})$, constant power, dried onto Whatman 3MM paper and are exposed to X-ray film for up to 15 hours.

Non-Denaturing Gels-Gels contain 5-12\% acrylamide (19:1, acrylamide:bisacrylamide). 3-6 $\mathrm{g}$ of DNA is suspended in $10-25 \mu \mathrm{L}$ of a solution of TAEMg. The solution is boiled and allowed to slowly cool to room temperature. Samples are then brought to a final volume of $20 \mu \mathrm{L}$ with a solution containing TAEMg, 50\% glycerol and $0.02 \%$ each of Bromophenol Blue and Xylene Cyanol FF tracking dyes. Gels are run on a Hoefer SE-600 gel electrophoresis unit at 10 Volts $/ \mathrm{cm}$ at $4 \mathrm{C}$ or at room temperature, and exposed to X-ray film for up to $15 \mathrm{hrs}$ or stained with Stainsall dye. Mobilities are measured relative to the Xylene Cyanol FF tracking dye.

\section{RESULTS}

\section{Assembly of junctions}

Demonstrating successful construction of structures as large as these is not readily amenable to the sorts of characterization available to smaller systems, such as NMR or X-ray crystallography; likewise, they are too small to be analyzed meaningfully by atomic force microscopy, as their largest features are of the order of the working resolution of typical AFM measurements. Here we use the techniques worked out previously to establish the formation of 3-arm, 4-arm, 5-arm and 6-arm junctions. These methods include [1] nondenaturing gel electrophoresis to demonstrate stability and estimate mass; [2] Ferguson analysis to see whether there is any suggestion of self-occlusion; and [3] hydroxyl radical autofootprinting to confirm the double helical nature of each arm, and to establish the presence or absence of base stacking at the branch point.

The essence of junction design is to put one part of a strand in one double helical arm and the rest of the strand in an adjacent arm; equal lengths for the two parts of each strand are not necessary, of course, but it is convenient to use them. 3-arm and 4-arm junctions form readily from 16-mer oligonucleotides, but we found that longer strands were needed to produce 5 -arm and 6-arm junctions. ${ }^{9}$ The minimum length strand needed to form a particular junction is not a firm number, but will be related to the sequences used and to the ionic strength of the solution. Given the success of using 16-mer arms (32-mer strands) to form 6-arm junctions, it seemed reasonable to try to use them to produce 8 -arm junctions. Figure 2a is a non-denaturing gel that demonstrates that this supposition proved to be correct. In structural DNA nanotechnology, the presence of a single target band in the vicinity of the expected molecular weight is usually accepted as convincing evidence for the stable formation of the target material. ${ }^{12}$ Here, we see that the band in lane 5 is in the vicinity of the expected size (128 nucleotide pairs), and that there are neither bands of higher mobility indicating breakdown/non-formation of the complex, nor bands of lower mobility indicating multimerization. A counterexample of the former criterion (i.e., failure indicated by breakdown) is seen below, and counterexamples showing multimerization are evident in refs. 13 and 14.

In contrast to the successful electrophoresis result shown in Figure 2a, Figure $2 b$ shows that a 12-arm junction designed with 16-nucleotide pairs per arm is insufficient to counteract all of the forces that oppose formation of the junction. This failure is demonstrated by the presence of breakdown products in lane 5; in addition to the target band, a number of other bands of higher mobility are visible, indicating breakdown of the product. In addition to the target complex (lane 5), mixtures of various junction subcomponents all contain breakdown products: strands 1 through 5 (lane 3), strands 1 through 6 (lane 4), and strands 7 through 12 (lane 6). Thus, more nucleotide pairs per arm are required if the number of arms is increased from 8 to 12 . In the same way that the arm-lengths were increased from 8 to 16 nucleotide 
pairs to form stable 5-arm and 6-arm junctions, ${ }^{9}$ the arm lengths here were increased from 16 to 24 nucleotide pairs to produce stable 12 -arm junctions. Figure $2 \mathrm{c}$ shows that the target material and some of its sub-complexes are all formed stably, according to the criteria above. Figure $2 \mathrm{~d}$ shows that all of the full junctions used in this study form stable complexes, as evidenced by single bands.

\section{Ferguson analysis}

It is worthwhile to establish the electrophoretic behavior of these junctions and to compare them with junctions containing fewer arms: We have determined the Ferguson ${ }^{21}$ plots of the 8 -arm junction by comparing its mobility with that of the mobilities of junctions containing 3 through 6 arms, as a function of polyacrylamide concentration. All junctions contain 16 nucleotide pairs per arm.

The mobility, M, of a molecule as a function of total gel concentration, T, may be described by the well-known relationship, ${ }^{22}$

$$
\log (\mathrm{M})=\log \left(\mathrm{M}_{\mathrm{o}}\right)-\mathrm{K}_{\mathrm{R}} \mathrm{T}
$$

where $\mathrm{M}_{\mathrm{o}}$ is the free mobility, and $\mathrm{K}_{\mathrm{R}}$ is the retardation coefficient. Rodbard and Chrambach 23 have shown that $K_{R}$ is an approximately linear function of the surface area (friction constant) of the electrophoresing species. If one models an $\mathrm{N}$-arm junction as a group of $\mathrm{N}$ cylinders emanating from a central site, the surface area of a series of junctions containing an increasing number of arms of identical length will increase linearly, as cylinders are added. Figure 3 a illustrates $\mathrm{K}_{\mathrm{R}}$ as a function of $\mathrm{N}$ for branched junctions with 16 nucleotide pairs per arm. The linear dependence seen, when $\mathrm{N}=3,5,6$, and 8 is in agreement with this model. The lower value of $K_{R}$ for the 4-arm junction (not included in the fit) is in agreement with experiments suggesting that its arms partially occlude each other ${ }^{20,24}$ thereby decreasing its overall effective surface area. This effect is maximized at $4{ }^{\circ} \mathrm{C}$, where the observed increase in friction constant is about 38\%, compared with $23 \%$ at $25^{\circ} \mathrm{C}$. Thus, there is a preferred pair of stacking domains for both domains of the 4-arm junction, which is well known. ${ }^{25,26}$ This double stacking dominance seems to be unique to the 4-arm junction.

A similar plot is shown in Figure 3b, for junctions containing 24 nucleotide pairs per arm. Again, the 4-arm junction shows a uniquely low friction constant. When excluded from the plot, its friction constant is $35 \%$ lower than expected at $4^{\circ} \mathrm{C}$. It is $16 \%$ lower at $25^{\circ} \mathrm{C}$, again showing that higher temperatures can obscure this effect. Thus, the 24-nucleotide-arm junctions behave similarly to 16-nucleotide junctions, although the impact of the stacking dominance in 4-arm junctions is seen to be slightly smaller, and significantly dependent on temperature.

\section{Hydroxyl radical analysis}

Churchill et al. ${ }^{20}$ first analyzed the structure of branched junctions in solution by means of hydroxyl radical autofootprinting experiments, in which the hydroxyl radicals have been generated by Fenton chemistry involving Fe(II)EDTA ${ }^{2-.19,27}$ This is a very powerful technique, because it gives single-nucleotide resolution of nucleoside susceptibility to attack. It produces a quantitative pattern of backbone cleavage at all possible sites. There is no requirement that the cleavage site be single-stranded. Duplex DNA is quite susceptible to hydroxyl radicals. 
The strategy of such experiments is to compare the chemical attack pattern of each strand when it is part of a junction, with the pattern obtained when the strand is complexed with its normal Watson-Crick complement. ${ }^{12}$ Experiments with 4 -arm junctions indicate that the patterns of two non-contiguous strands are the same in both environments, while the other two strands, those that cross over at the branch point, exhibit protection at the site of the junction. From those experiments it was concluded that strands with the same patterns in both pairing environments probably have double helical conformations near the junction, while the other strands form crossover structures. ${ }^{20}$ The arms shared by helical strands are thought to form a continuously stacked helical domain within the junction structure, so, as noted above, the 4-arm junction contains two helical domains. Crossover points and occluded points have evinced protection in virtually all analyses of unusual motifs. ${ }^{13,14}$ By contrast, the hydroxyl radical autofootprint of the 5-arm junction examined earlier showed protection on all strands at the branch point, suggesting no dominant stacking pattern. ${ }^{9}$ The 6-arm junction in the same study showed a single example of stacking dominance: One strand had the same pattern as in the double helical baseline control strand, suggesting stacking between the two helices in which the strand partook. This result was confirmed by the resistance of this strand to cleavage by the resolvase endo VII, unlike the other five strands in the junction. ${ }^{9}$

Thus, the autofootprinting patterns of the 8-arm junction and the 12-arm junction were obtained, because they would be likely to reveal any stacking dominance or other unusual features that might exist in these junctions. In addition, they confirm the double helical nature of the arms of the junction. We regard a nucleotide as protected if its intensity is lowered relative to its neighbors as one compares the junction to the double strand. The autofootprint for the 8-arm junction is shown in Figure 4a. In each case, there appears to be protection of one or both of the junction-flanking nucleotides, relative to the double helical baseline pattern. Thus, we conclude that there is no dominant stacking domain in the 8-arm junction. The autofootprint for the 12-arm junction is shown in Figure 4b. Again, every strand is protected at its junction-flanking sites. Thus, neither of the junctions demonstrates the stacking dominance that characterizes the 4-arm junction and one of the strands of the previously studied 6-arm junction.

The protection patterns of each strand distant from the branch point resembles closely the protection pattern of the same strand in the double stranded control. This finding suggests strongly that the individual strands have formed a set of double helical arms, thus producing the target junctions. By contrast with the 4-arm junction ${ }^{20}$ and with one pair of arms in the previously characterized 6-arm junction, ${ }^{9}$ neither the 8 -arm junction nor the 12 -arm junction shows any preferred stacking amongst its arms.

No significant protection is seen at nucleotides well beyond the junction-flanking positions, suggesting a lack of branch migration in all cases. In the well-characterized 4-arm junction $\mathrm{J} 1,{ }^{20}$ some protection was noted at the nucleotide $5^{\prime}$ to the junction flanking position in the crossover strands. This is seen at position 15 of strands 3 and 6 of JXW8G and position 23 in strands 6 , strand 9 of JXW12O. Protection on the $3^{\prime}$ nucleotide is present at position 18 in strands 6, 7 and 8 of JXW8G, and at position 26 of strands 2 and 6 of JXW12O. Position 26 on strands 7 and 10 is protected relative to position 27 in the double strand, but is strongly protected in the junction. It is hard to interpret these protections, but they suggest that the domains are not well-stacked at these nucleotides in the vicinity of the junction. There is nothing to suggest from these patterns that isomerizations akin to branch migration occur. 


\section{DISCUSSION}

\section{Stability and Structure of 8-Arm and 12-Arm Junctions}

We have demonstrated that it is possible to construct the target molecules by the following experiments: Non-denaturing gel electrophoresis has shown that we can produce robust constructs from both 8-strand and 12-strand components. We find that it is possible to design and construct 8 -arm junctions from strands containing 32 nucleotides (16 nucleotide pairs per arm), but 48 nucleotides ( 24 nucleotide pairs per arm) are needed to build 12strand complexes that produce a single band on a gel. The similarity of the hydroxyl radical protection patterns of both double stranded DNA and the strands of each junction far from the branch point confirms the notion that all strands are present in double helices, both before and after the branch point.

Successful construction of the 8-arm junctions demonstrates that contiguous base pairs can be placed in all the junction-flanking nucleotides completely around the junction. In addition, hydroxyl-radical autofootprinting shows that junctions that do not branch migrate can be built, even if they contain more than 8 arms. ${ }^{28}$ The principle used here to produce the 12 -arm junction, repeating the same junction-flanking nucleotide sequence three times around the center (Figure 1), appears to be effective for a stable junction. This finding validates the hypothesis of widely separated identical base pairs around the junction. ${ }^{28}$ Here, we maximized the separation of identical pairs by distributing them symmetrically. We see no evidence that 12 -arms is the maximum number of arms possible for a junction. For example, if an application were to arise that involved solving graph-theory problems using DNA-based computation ${ }^{7}$ that required more arms, it likely would be possible to build the needed constructs. In larger junctions, it might not be necessary to maximize separation as we have done here.

We have noted that longer arms are needed to stabilize the 12-arm junction. One might think that this change is necessary to counteract the charge at the center of the junction; however, in moving from an 8 -arm junction to a 12 -arm junction, one is not cramming more charge into the same volume, but, rather, is expanding the effective size of the central portion of the junction. Our hydroxyl radical autofootprinting data show that the 24-nucleotide arms are quite capable of sustaining the stacking dominance seen in shorter 4-arm junctions; however our data show that the junctions constructed here with 8-arms and 12-arms do not display this feature to any extent at all, in contrast to the previously examined 6-arm junction. ${ }^{9}$

The Ferguson analysis experiments speak to the tertiary structure of the junctions. The agreement of the model of the increase of friction constants with increasing number of arms suggests that all arms are equally accessible to the acrylamide gel matrix. This finding would be compatible with a number of different tertiary structures for these species. The planar structures shown in Figure 1 are only conveniences for illustrating connectivity. It is likely that the arms will minimize electrostatic repulsion by assuming some sort of 3D tertiary structure. Thus, it is more likely that the 8-arm junction arranges the tips of its arms at the corners of a cube than of a stacked tetragonal prism, as suggested by the hydroxyl radical autofootprinting (no stacking at the junctions) and by the Ferguson analysis (no occluded helices). From the same analysis of the 12-arm junctions, the data suggests that it is more likely that the tips of the 12-arm junction are assorted to sit at the vertices of an icosahedron than of a hexagonal prism. However, these are just suggestions of likely trends; detailed structural modeling is well beyond the scope of this work.

\section{Macromolecular Construction with 8-Arm and 12-Arm Junctions}

The ability to construct 8-arm and 12-arm junctions means that a large number of structures can be constructed from branched DNA molecules that were previously impossible. 
Williams ${ }^{29}$ has tabulated the space-filling networks with 432 symmetry that can be formed from Platonic and Archimedean solids. These structures can, in principle, be formed from the helix axes of DNA molecules; the symmetry is much lower if one considers the entire DNA molecule. Figure 5a illustrates an 8-connected network based on the packing of cuboctahedra (red) and octahedra (blue). Each vertex is connected to four edges that form two sides of osculating coplanar squares (say the tops of the cuboctahedra on the lower right of Figure 5a), and four others that come from another pair of squares perpendicular to the first pair. The cavities are filled by octahedra.

Figure $5 \mathrm{~b}$ illustrates the basis for the stability of cubic close packing (face centered cubic packing) which is a 12-connected network. This basis is shown as Buckminster Fuller's 'octahedral truss.' ${ }^{10}$ Two edge-sharing octahedra (blue) could wobble back and forth about their common edge, but are kept from wobbling by the truss, drawn in red. This leads to the formation of a tetrahedron, indicated with shaded faces. Figure $5 \mathrm{c}$ shows a view down the common edge (a magenta dot), illustrating how the truss (top and bottom) could keep a lattice of octahedra from wobbling. Figure $5 \mathrm{~d}$ shows a top view of nine octahedra. Four of the tetrahedra are drawn with thicker edges (red on top, magenta on the bottom and blue on the sides. The vertices of these tetrahedra are emphasized with black dots to indicate their 12-connected character. Each vertex has four upward-directed edges (forming the bottom of an octahedron above), four downward-directed edges (forming the top of an octahedron below) and four coplanar edges corresponding to the shared edges of four octahedra (as in Figure 5b).

The issue of chirality remains for any branched junction with more than 3 arms. However, the recent success of Turberfield and his colleagues ${ }^{30}$ in forming tetrahedra of only a single chirality suggests that the asymmetries of nucleic acid double helices may solve this problem. Another issue is whether it is more effective to build N-connected lattices of the sort shown in Figure 5 (or even simpler lattices) from $\mathrm{N}$-arm branched junctions, or whether it is more useful to use edge-sharing cohesion $^{31}$ for this purpose. The answer will require further experimentation, but the existence of 8-arm and 12-arm junctions will enable this determination to be made.

\section{Supplementary Material}

Refer to Web version on PubMed Central for supplementary material.

\section{Acknowledgments}

This research has been supported by grants GM-29554 from the National Institute of General Medical Sciences, grants DMI-0210844, EIA-0086015, CCF-0432009, CCF-0523290 and CTS-0548774, CTS-0608889 from the National Science Foundation, 48681-EL from the Army Research Office, DE-FG02-06ER64281 from the Department of Energy (Subcontract from the Research Foundation of SUNY), NTI-001 from Nanoscience Technologies, Inc and a grant from the W.M. Keck Foundation.

\section{References}

1. Kornberg, A. DNA Replication. W.H. Freeman; New York: 1980.

2. Holliday R. Genet Res. 1964; 5:282-304.

3. Seeman NC. Nature. 2003; 421:427-431. [PubMed: 12540916]

4. Seeman NC, Lukeman PS. Reports Prog Phys. 2005; 68:237-270.

5. Chen J, Seeman NC. Nature. 1991; 350:631-633. [PubMed: 2017259]

6. Zhang Y, Seeman NC. J Am Chem Soc. 1994; 116:1661-1669.

7. Sa-Ardyen P, Jonoska N, Seeman NC. J Am Chem Soc. 2004; 126:6648-6657. [PubMed: 15161292] 
8. Panyutin IG, Hsieh P. J Mol Biol. 1993; 230:413-424. [PubMed: 8464057]

9. Wang Y, Mueller JE, Kemper B, Seeman NC. Biochem. 1991; 30:5667-5674. [PubMed: 1645997]

10. Edmondson, A. A Fuller Explanation. Birkhauser; Boston: 1987.

11. Cantor CR, Schimmel PB. Biophysical Chemistry. 1980; 3:1205-1209.

12. Seeman, NC. Curr Protocols Nucl Acid Chem. John Wiley \& Sons; New York: 2002.

13. Fu TJ, Seeman NC. Biochem. 1993; 32:3211-3220. [PubMed: 8461289]

14. Du SM, Zhang S, Seeman NC. Biochem. 1992; 31:10955-10963. [PubMed: 1332747]

15. Seeman NC. J Biomol Str \& Dyns. 1990; 8:573-581.

16. Kallenbach NR, Ma RI, Seeman NC. Nature. 1983; 305:829-831.

17. Caruthers MH. Science. 1985; 230:281-285. [PubMed: 3863253]

18. Maxam AM, Gilbert W. Proc Nat Acad Sci (USA). 1977; 74:560-564. [PubMed: 265521]

19. Tullius TD, Dombroski B. Science. 1985; 230:679-681. [PubMed: 2996145]

20. Churchill MEA, Tullius TD, Kallenbach NR, Seeman NC. Proc Nat Acad Sci (USA). 1988; 85:4653-4656. [PubMed: 3387432]

21. Ferguson KA. Metabolism. 1964; 13:985-1002. [PubMed: 14228777]

22. Rodbard D, Chrambach A. Proc Natl Acad Sci (USA). 1970; 65:970-977. [PubMed: 4191703]

23. Rodbard D, Chrambach A. Anal Biochem. 1971; 40:95-134. [PubMed: 5550151]

24. Kimball A, Guo Q, Lu M, Kallenbach NR, Cunningham RP, Seeman NC, Tullius TD. J Biol Chem. 1990; 265:6544-6547. [PubMed: 2157702]

25. Lilley DMJ, Clegg RM. Ann Rev Biophys \& Biomol Str. 1993; 22:299-328.

26. Seeman NC, Kallenbach NR. Ann Rev Biophys \& Biomol Str. 1994; 23:53-86.

27. Tullius TD, Dombroski B. Proc Natl Acad Sci (USA). 1986; 83:5469-5473. [PubMed: 3090544]

28. Seeman, NC.; Kallenbach, NR. Molecular Structure: Chemical Reactivity and Biological Activity. Stezowski, JJ.; Huang, J-L.; Shao, M-C., editors. Oxford University Press; Oxford: 1988. p. 189-194.

29. Williams, R. The Geometrical Foundation of Natural Structure. Dover; NewYork: 1979.

30. Goodman RP, Schaap IAT, Tardin CF, Urban CM, Berry RM, Schmidt CF, Turberfield AJ. Science. 2005; 310:1661-1665. [PubMed: 16339440]

31. Yan H, Seeman NC. J Supramol Chem. 2001; 1:229-237. 

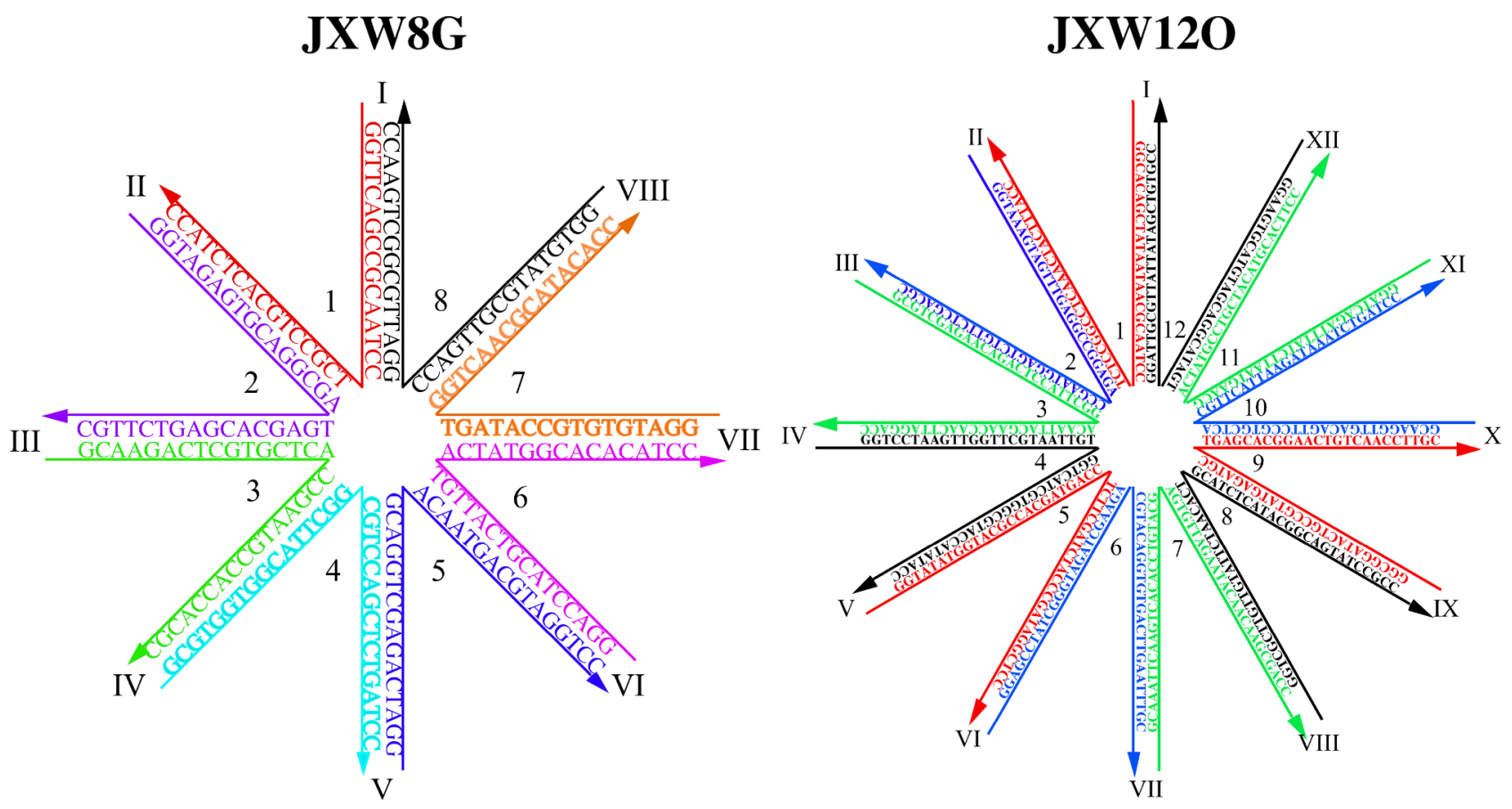

Figure 1.

The Sequences of the Junctions Constructed in these Experiments. The 8-arm junction, JXW8G is shown on the left, and the 12-arm junction, JXW12O is shown on the right. The color code for the 8-arm junction is arbitrary, but that of the 12-arm junction is designed to show that the junction flanking sequences are the same every four arms. Regardless of this aspect of sequence symmetry, the junctions do not appear to undergo branch migration. 
(a)

(c)

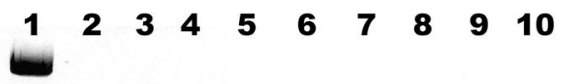

25 bp

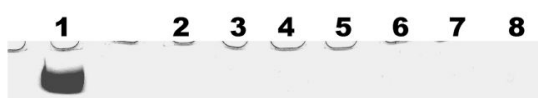

(b)

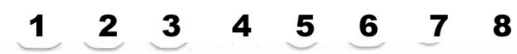

200 bp

$125 \mathrm{bp}$
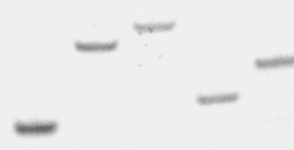

$300 \mathrm{bp}$

250 bp

200 bp

125 bp (d)

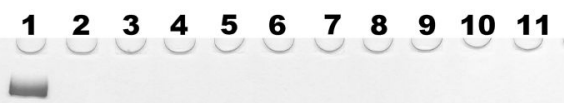

$300 \mathrm{bp}$

200 bp

$125 \mathrm{bp}$
Figure 2.

Non-Denaturing Gels demonstrating the stabilities of the junctions. All gels are 5\% nondenaturing polyacrylamide gels stained with Stainsall dye. (a) Electrophoresis of equimolar ratios of JXW8G. The finial concentration for each lane is $1 \mu \mathrm{M}$. Lane 1 contains a 25 base pair marker wherein the darker band indicates 125 nucleotide pairs DNA; this marker is used in lane 1 in all panels. Lanes $2-8$ contain each of the following strands: (2) $1+2$; (3) $1+2+3+4$; (4) $1+2+3+4+5+6+7$; (5) all strands of JXW8G; (6) $1+2+3+4+5$; (7) $1+2+3+4+5+6$; (8) $1+2+3$. Note in particular that the full junction in lane 5 is stable, with only a single band present. (b) Electrophoresis of equimolar ratios of a 12-arm junction containing 16 nucleotide pairs per arm (JXW12G). Lanes $2-8$ contain the following strands: (2) $1+2+3+4$; (3) $1+2+3+4+5$; (4) $1+2+3+4+5+6$; (5) all strands of JXW12G; (6) 
$12+11+10+9+8+7 ;$; 7 ) $11+10+9+8+7$; (8) $10+9+8+7$. Several of the lanes, including the full junction lane, indicate breakdown. (c) Electrophoresis of equimolar ratios of the 12-arm junction, JXW12O. The finial concentration for each lane is $1 \mu \mathrm{M}$. Lanes $2-10$ contain the following strands: (2) $1+2+3+4$; (3) $1+2+3+4+5+6$; (4) $1+2+3+4+5+6+7+8$; (5) $1+2+3+4+5+6+7+8+9+10 ;(6)$ all strands of JXW12O; (7) $1+2+3+4+5+6+7+8+9+10+11$; (8) $1+2+3+4+5+6+7+8+9$; (9) $1+2+3+4+5+6+7$; (10) $1+2+3+4+5$. Note in particular that the full junction in lane 6 is stable, with only a single band present. (d) Electrophoresis of all junctions used in electrophoresis studies. The lanes contain (2): JYG (3-arm junction with 16-nucleotide arms); (4) 3: JXG (4-arm junction with 16-nucleotide arms); (4): JYL5G (5arm junction with 16-nucleotide arms); (5): JYL6G (6-arm junction with 16-nucldotide arms); (6): JXW8G (8-arm junction in Figure 1); (7): JXW12O (12-arm junction in Figure 1); (8): JXW6O (6-arm junction with 24-nucleotide arms); (9): JXW5O (5-arm junction with 24-nucleotide arms); (10): JXO (4-arm junction with 24-nucleotide arms); (11): JYO, (3-arm junction with 24-nucleotide arms). All are well-behaved, migrating as a single band. 

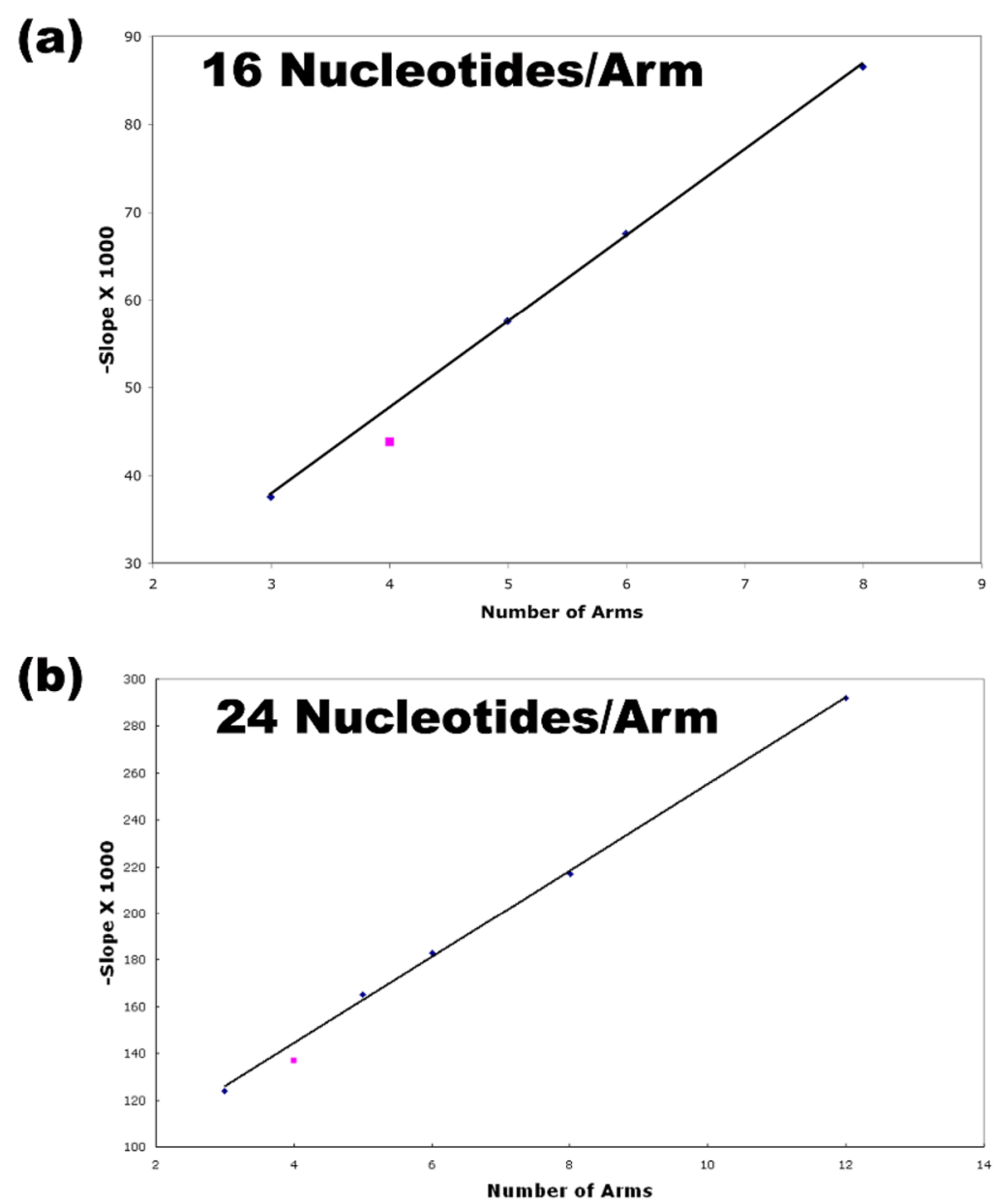

Figure 3.

Ferguson Slopes of the Junctions Built Here and Related Junctions. (a) Ferguson slopes of 3arm, 4-arm, 5-arm, 6-arm, and 8-arm junctions (each arm contains 16 nucleotide pairs). Gels were run at 4 degrees. The Ferguson slopes of the junctions, are as follows: JYG (0.038), JXG (0.044), JYL5G (0.058), JYL6G (0.068), JXW8G (0.087). The line was fitted excluding the four-arm junction, whose position was then plotted. The increment for JXG is about $38 \%$ lower than expected at 4 degrees, on the basis of the relationship between the other four junctions. (b) Ferguson slopes of 3-arm, 4-arm, 5-arm, 6-arm, 8-arm, and 12-arm junctions. Gels were run at 4 degrees. The Ferguson slopes of the junctions, given as -slope, are as follows: JYO (0.124), JXO (0.137), JXW5O (0.165), JXW6O (0.183), JXW8O (0.217), JXW12O (0.292). The line was fitted excluding the four-arm junction, whose position was then plotted. The increment for JXO is about $35 \%$ lower than expected at 4 degrees, on the basis of the relationship between the other five junctions. 
(a)

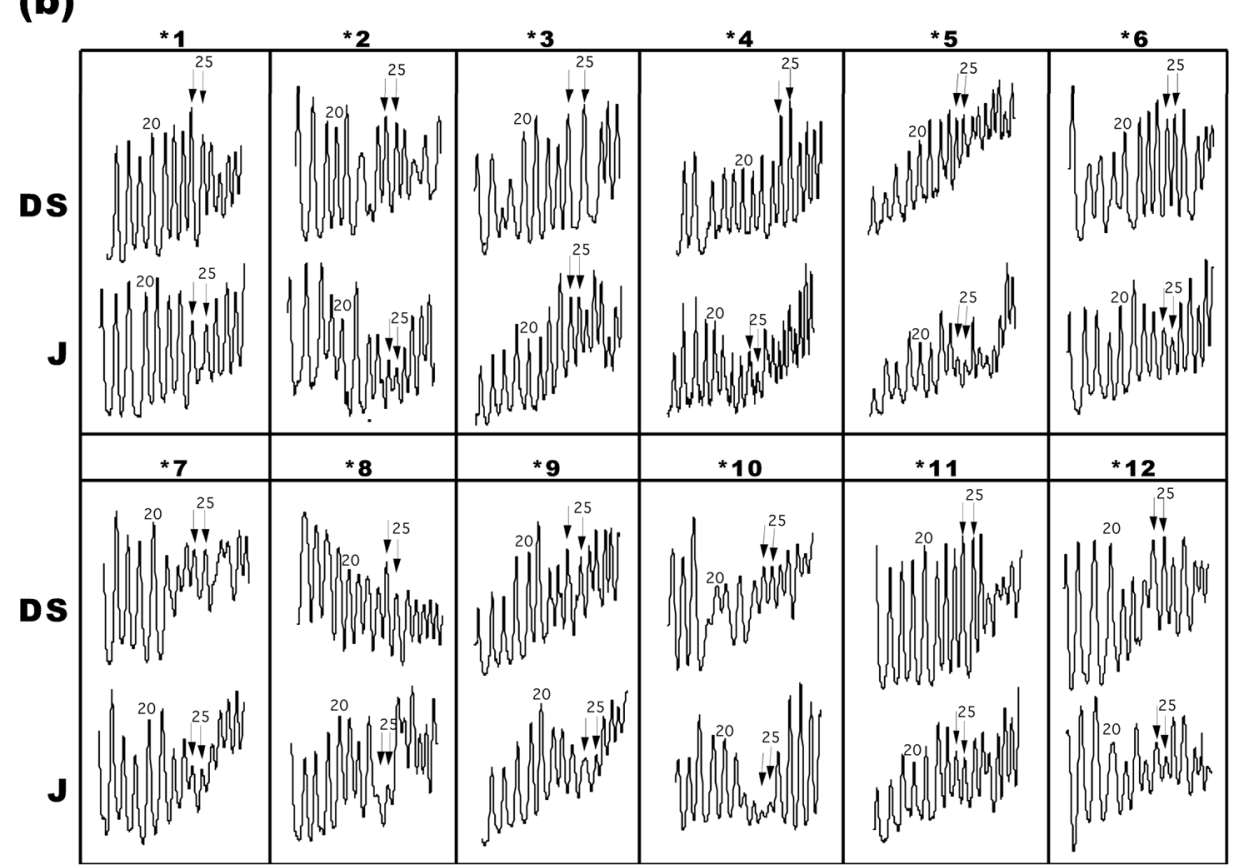

Figure 4.

Hydroxyl Radical Cleavage of 8-Arm and 12-Arm Junctions. The same conventions apply to both panels. 'DS' represents the cleavage pattern of a particular labeled strand (indicated by $* \mathrm{n}$, where $\mathrm{n}$ is the number of the strand) when it is in a double stranded complex opposite its Watson-Crick complement. 'J' represents the cleavage pattern of the same strand when in the junction complex. Nucleotide numbering is indicated over the patterns. The junctionflanking residues are indicated by a pair of arrows. (a) The patterns obtained from the 8-arm junction. (b) The patterns obtained from the 12-arm junction. In both cases, the junctionflanking nucleotides are protected in the J pattern, relative to the DS pattern. 
(a)

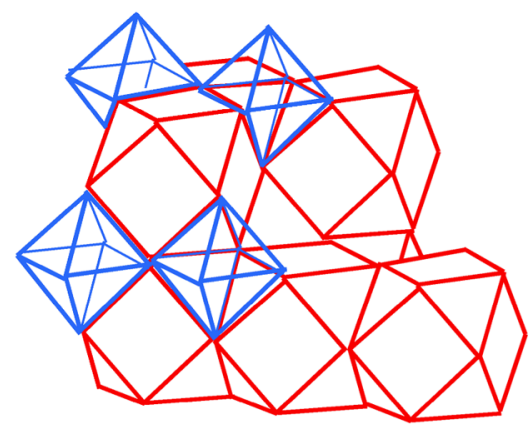

(c)

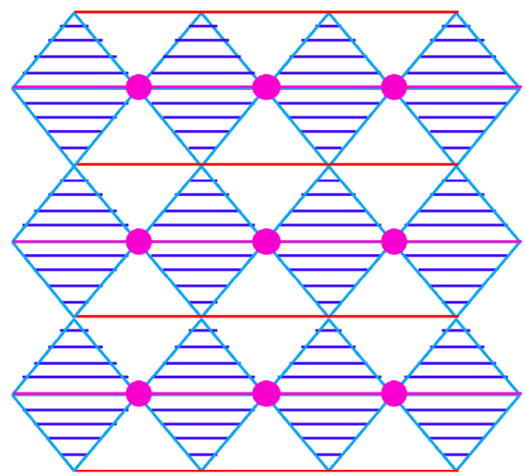

(b)

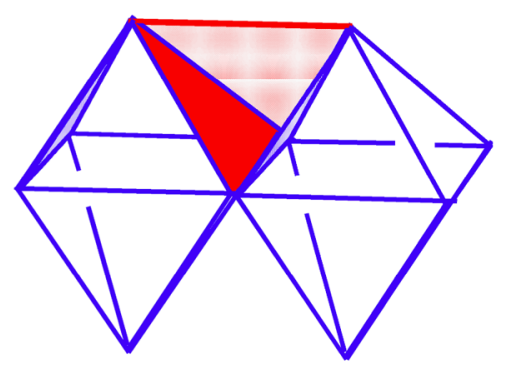

(d)

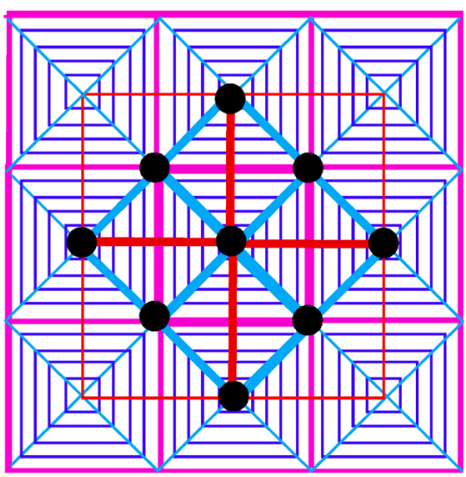

Figure 5.

N-Connected Networks Enabled by the Existence of 8-Arm and 12-Arm Junctions. (a) An 8Connected Network. A series of cuboctahedra sharing their square faces are drawn in red. The cavities created by this arrangement can be filled by octahedra, drawn in blue. (b) The Fuller Octahedral Truss. Two octahedral share an edge, about which they could wobble. However, their upper vertices are joined by an added red edge (the truss), which keeps them from wobbling. This creates a tetrahedron, shown with three of its faces shaded. (c) shows an array of octahedra looking down the shared edge, indicated as a magenta dot. The truss is also shown, again in red. The tetrahedra are clear in this projection. (d) shows a top view of the 12-connected face-centered cubic-close packing lattice that this arrangement generates. Nine octahedra are shown, outlined in magenta squares. The vertices of the octahedra closest to the reader are the centers of concentric blue squares. The trusses are drawn again in red. The four tetrahedra at the center are drawn with thicker lines for their edges; each has a red (truss) edge nearest the viewer, four blue edges further away, and a magenta edge furthest from the viewer. Nine of the 12-connected vertices are emphasized by black dots. Five of these emphasized vertices are at the tops of octahedra (on the red lines), and four are at the bottom edges of the tetrahedra. However, all have the same environments in the full lattice. 
Design Molecules

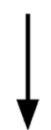

Do Complexes Form?

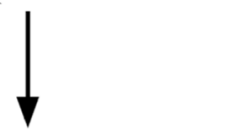

Single Band on Non-Denaturing Gel No

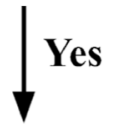

Ferguson Plot

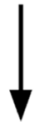

Non-Linear Friction Constant Plot if Helix-Helix Occlusion (No).

Hydroxy Radical Autofootprint

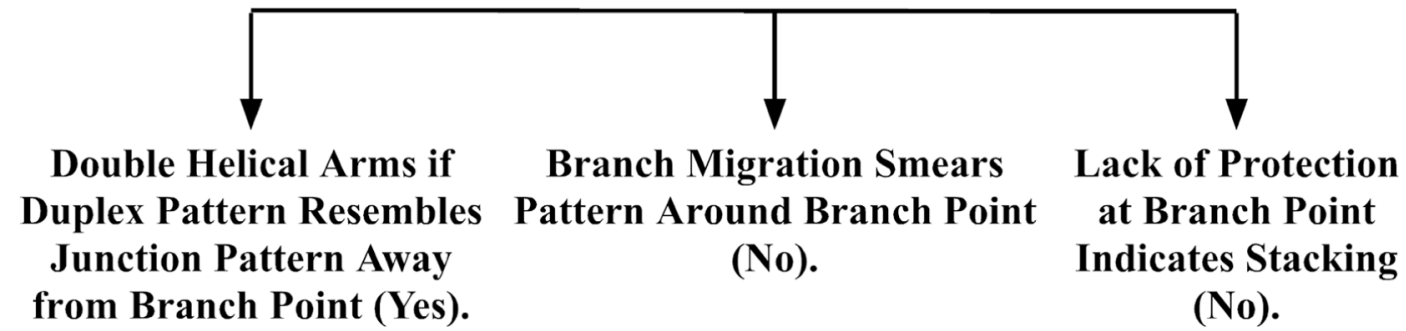

Scheme 1.

The analytical scheme used to demonstrate and to characterize the 8-arm and 12-arm junctions built here. The flow chart at top is used to establish if the junction has formed properly; the 8-arm junction yielded a 'Yes' with the first design (16 nucleotide pairs/arm), but the 12-arm junction required a second design increasing the number of nucleotide pairs/ arm from 16 to 24 . The part of the scheme below that describes characterization. '(Yes)' and '(No)' indicate the answers for both junctions. 